\title{
ADSC-conditioned media elicit an ex vivo anti-inflammatory macrophage response
}

\author{
Maria Jacoba Kruger, Maria Martha Conradie, Magda Conradie and Mari van de Vyver \\ Division of Endocrinology, Department of Medicine, Faculty of Medicine and Health Sciences, Stellenbosch University, Cape Town, South Africa
}

Correspondence should be addressed to M van de Vyver: vandevyverm@sun.ac.za

\begin{abstract}
Obesity-associated inflammatory mechanisms play a key role in the pathogenesis of metabolic-related diseases. Failure of anti-inflammatory control mechanisms within adipose tissue and peripheral blood mononuclear cells (PBMCs) have been implicated in disease progression. This study investigated the efficacy of allogeneic adipose tissuederived mesenchymal stem cells conditioned media (ADSC-CM) to counteract persistent inflammation by inducing an anti-inflammatory phenotype and cytokine response within PBMCs derived from patients with and without metabolic syndrome. Forty-six ( $n=46)$ mixed ancestry females (18-45 years) were subdivided into (a) healthy lean (HL) $(n=10)\left(\mathrm{BMI}<25 \mathrm{~kg} / \mathrm{m}^{2}\right)$, (b) overweight/obese (OW/OB) (BMI $\geq 25 \mathrm{~kg} / \mathrm{m}^{2},<3$ metabolic risk factors) ( $n=22$ ) and (c) metabolic syndrome (MetS) (visceral adiposity, $\geq 3$ metabolic risk factors) ( $n=14)$ groups. Body composition (DXA scan), metabolic (cholesterol, HDL, LDL, triglycerides, blood glucose) and inflammatory profiles (38-Plex cytokine panel) were determined. PBMCs were isolated from whole blood and treated ex vivo with either (i) autologous participant-derived serum, (ii) ADSCs-CM or (iii) a successive treatment regime. The activation status (CD11b+) and intracellular cytokine (IL6, IL10, TNFa) expression were determined in M1 (CD68+CD206-CD163-) and M2 (CD68+CD163+ CD206+) macrophage populations using flow cytometry. ADSC-CM treatment, promoted a M2 macrophage phenotype and induced IL10 expression, this was most pronounced in the OW/OB group. This response is likely mediated by multiple complementing factors within ADSC-CM, yet to be identified. This study is the first to demonstrate the therapeutic potential of ADSC-CM to restore the inflammatory balance in immune compromised obese individuals.
\end{abstract}

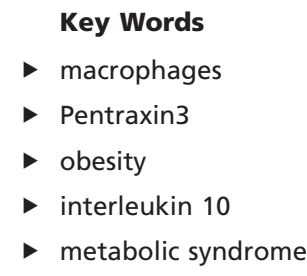

Journal of Molecular Endocrinology (2018) 61, 173-184

\section{Introduction}

Obesity-associated inflammatory mechanisms play a key role in the pathogenesis of metabolic-related diseases such as metabolic syndrome and type 2 diabetes (Esser et al. 2014). Disruption of normal endocrine function affects glucose homeostasis and occurs as a result of changes within the adipose tissue cellular composition (Esser et al. 2014, Martyniak \& Masternak 2017, Pirola \& Ferraz 2017). This is characterised by adipocyte hypertrophy and the formation of crown-like structures due to excessive infiltration and proliferation of inflammatory macrophages (Esser et al. 2014, Braune et al. 2017, Martyniak \& Masternak 2017, Pirola \& Ferraz 2017). The infiltrating macrophages derive primarily from peripheral blood mononuclear cells (PBMCs) and can have either a pro- (M1) or anti- (M2) inflammatory phenotype (Bories et al. 2012, Dey et al. 2014, Braune et al. 2017). 
Inflammatory dysregulation during obesity has been linked to alterations in the functional programming of PBMCs and the ratio of M1 to M2 macrophages within the PBMC population (Bories et al. 2012, Esser et al. 2014).

The immunomodulatory and paracrine properties of mesenchymal stem cells (MSCs) have the potential to regulate multiple signalling pathways that contribute to the pathogenesis of chronic inflammation and hyperglycaemia (Chen et al. 2016, Fontaine et al. 2016, Zachar et al. 2016, Harting et al. 2018). A reduction in the size of the circulating stem/progenitor cell pool within the PBMC population is however evident with disease progression and corresponds with the severity of secondary complications (Egan et al. 2008, Fadini et al. 2017, Rigato \& Fadini 2017). Intravenous infusion of MSCs therefore presented as a novel strategy to alleviate inflammation, hyperglycaemia and its associated microvascular complications. Although the safety and toxicity of intravenously infused human adipose tissuederived MSCs have been investigated in both mouse models and patient-based studies (Ra et al. 2011a,b, Toupet et al. 2013), the long-term tumourigenicity of infused cells remains a concern. A further limiting factor is the unpredictability of autologous cell therapy, especially in patients that suffer from advanced metabolic dysfunction. As a consequence of the in vivo pathological niche microenvironment, the paracrine signalling response of endogenous MSCs becomes impaired and compromises their therapeutic potential (Boland et al. 2017, van de Vyver 2017). In a co-culture experiment that mimic the diabetic microenvironment, Boland et al. (2017) recently demonstrated that in this simulated environment, MSCs not only lose their ability to suppress inflammation but actively enhance inflammation through induction of pro-inflammatory cytokine secretion by PBMCs. To avoid adverse effects associated with unpredictable cellular responses, it is hypothesised that allogeneic MSC-derived conditioned media could potentially be used as an alternative to cell therapy.

In this study, we investigated the efficacy of allogeneic MSC-derived conditioned media to counteract persistent inflammation by inducing an anti-inflammatory phenotype and cytokine response within PBMCs derived from patients with and without metabolic syndrome.

\section{Materials and methods}

Ethical approval was obtained from the Health Research Ethics committee at Stellenbosch University (N15/07/066) and permission was granted for the research to be conducted at Tygerberg Academic hospital from the Western Cape Department of Health (WC_2016RP14858). Volunteers were informed about the purpose and risks of the study before signing an informed consent form. All experimental procedures were conducted according to the ethical guidelines and principles of the Declaration of Helsinki.

\section{Participant recruitment and sample collection}

A total of forty-six ( $n=46)$ adult females (age 18-45 years) were included in the study. Volunteers that were either pregnant, breastfeeding, less than 10 months postpartum or that suffered from any infection or infectious disease were excluded from the study. Due to the demographics of the population surrounding Tygerberg hospital, the participants included in this study were of mixed ancestry. This specific ethnic population is known to have a genetic predisposition and especially females have a considerable risk to develop type 2 diabetes (Goedecke et al. 2017). The data presented in this study are thus specific for this population and should be interpreted as such.

Upon inclusion into the study, participants completed nutritional and lifestyle questionnaires, underwent a Dual Energy X-ray Absorptiometry (DXA) scan (body composition) (Hologic Discovery W, Serial Number 70215, Hologic Inc, Marlborough, USA) and anthropometrical measurements (height $(\mathrm{m})$, weight $(\mathrm{kg})$, hip circumference $(\mathrm{cm})$, waist circumference $(\mathrm{cm}))$. Fasting blood glucose (FBG) levels (finger prick) were determined using the Contour plus glucometer system (BAYER, Leverkusen, Germany) and whole blood samples were collected into EDTA and SST tubes (BD Vaccutainer, Franklin Lakes, NJ, USA).

The participants were subdivided into three groups based on their metabolic health. The traditional metabolic risk factors used for subdivision of the cohorts included: visceral adiposity (waist-to-hip-ratio $>0.85$ and trunkto-limb fat mass ratio $>1$ ), dyslipidaemia (triglycerides (TGS) $>1.7 \mathrm{mmol} / \mathrm{L}$ and high-density lipoprotein (HDL) $<1.29 \mathrm{mmol} / \mathrm{L}$ ), hypertension (systolic blood pressure (BP) $>130 \mathrm{mmHg}$ and diastolic BP $>85 \mathrm{mmHg}$ ) and hyperglycaemia (FBG $>5.6 \mathrm{mmol} / \mathrm{L}$ ). The criteria for each specific subgroup were as follows: (i) healthy lean (HL) (BMI $<25 \mathrm{~kg} / \mathrm{m}^{2}$, metabolically healthy) $(n=10)$; (ii) healthy overweight/obese (OW/OB) (BMI $\geq 25 \mathrm{~kg} / \mathrm{m}^{2}, \leq 2$ metabolic risk factors) $(n=22)$ and (iii) metabolic syndrome (MetS) (visceral adiposity, $>3$ metabolic risk factors) $(n=14)$. The criteria used to identify individuals with MetS complied with the guidelines provided by 
the International Diabetes Federation (www.IDF.org, Metabolic syndrome consensus statement 2005, accessed 10 June 2017).

\section{Serum sample processing and analysis}

Whole blood collected in SST tubes were left to clot at room temperature before centrifugation at $1500 \mathrm{~g}$ and $4^{\circ} \mathrm{C}$ for $10 \mathrm{~min}$. Serum was aliquoted and stored at $-80^{\circ} \mathrm{C}$ until subsequent analysis. Each participant's lipid profile (total cholesterol, TGS, HDL and low-density lipoprotein (LDL)) was determined using an Alere Afinion TM Lipid Panel, (\#10183107, Alere, Waltham, MA, USA) in combination with the Afinion AS100 Analyzer (Alere). Serum cytokine concentrations were quantified using a 37-Bio-Plex Pro Human inflammation cytokine assay kit (\#171-AL001M, Bio-Rad Laboratories Ltd.). The following analytes were assessed: a proliferation-inducing ligand (APRIL), also known as tumour necrosis factor ligand superfamily member 13 (TNFSF13); B-cell activating factor (BAFF) /TNFSF13B; soluble cluster of differentiation 30 (sCD30) /TNFRSF8; sCD163, Chitinase-3-like 1; the signaltransducing component gp130, also known as soluble interleukin-6 receptor B (sIL6RB); sIL6Ra; interferon (IFN)a2; IFNB; IFNy; interleukins (IL)-2, -8, -10, -11, -12 (p40 and p70 subunits), -19, -20, -22, -26, -27 (p28), $-28 \mathrm{~A} / \mathrm{IFN} \lambda 2,-29 /$ IFN $\lambda 1,-32,-34,-35$; LIGHT/TNFSF14; matrix metalloproteinases (MMP)-1, -2, -3; Osteocalcin; Osteopontin; Pentraxin3 (PTX3); soluble tumour necrosis factor receptors (sTNFR1 and sTNFR2); thymic stromal lymphopoietin (TSLP) and TNF-related weak inducer of apoptosis TWEAK/TNFSF12. Additionally, separate ELISA were used to quantify IL6 (E-EL-H0102, Elabscience Biotechnology Inc., Houston, TX, USA) and C-reactive protein (CRP) (E-EL-H5134, Elabscience Biotechnology Inc., Houston, TX, USA) levels within serum. Refer to Supplementary Table 1 (see section on supplementary data given at the end of this article) for the sensitivity levels for each of the analytes assessed.

\section{PBMC isolation, treatment and flow cytometry analysis}

PBMCs were isolated from the whole blood collected in EDTA tubes using density centrifugation. Whole blood was layered upon an equal volume of Histopaque-1077 (Sigma-Aldrich) and centrifuged at $400 \boldsymbol{g}$ for $30 \mathrm{~min}$ at room temperature. The opaque interface containing PBMCs was then transferred to a new tube, washed using PBS (centrifuged $250 \boldsymbol{g}, 10 \mathrm{~min}$ ) and resuspended in freezing media $(20 \%$ human serum albumin (Sigma-Aldrich), 10\% DMSO (Merck) and 70\% DMEM with ultra-glutamine (BioWittaker, Lonza, Basel, Switzerland)) before storage at $-80^{\circ} \mathrm{C}$.

PBMCs were rapidly thawed at $37^{\circ} \mathrm{C}$, centrifuged for $5 \mathrm{~min}$ at $400 \boldsymbol{g}$ and resuspended in DMEM with ultra-glutamine (BioWittaker) containing 1\% penicillin /streptomycin (BioWittaker) and 10\% foetal bovine serum (FBS) (Biochrom, Berlin, Germany). PBMCs at a concentration of $1 \times 10^{6}$ cells were treated with either (i) 20\% autologous participant-derived serum (4h); (ii) adipose-derived stem cell (ADSC)-conditioned media (CM) (4h) or (iii) with successive treatment of $20 \%$ autologous participant-derived serum $(4 \mathrm{~h})$ followed by ADSC-CM (4h). In the successive treatment, PBMCs were therefore primed with autologous serum (representative of the in vivo environment after thawing) prior to treatment with ADSC-CM. The priming (serum) and treatment (ADSC-CM) times were kept consistent ( $4 \mathrm{~h}$ each).

Following treatment, PBMCs were washed, resuspended in $50 \mu \mathrm{L}$ staining buffer (PBS containing $20 \%$ FBS, filtered) and co-labelled with surface marker antibodies (4 $\mu \mathrm{L} \quad$ CD68-BV421 (BD564943), $\quad 4.4 \mu \mathrm{L}$ C11b-APC-Cy7 (BD557754), 4.2 $\mu \mathrm{L}$ CD163-PERCP-Cy5.5 (BD563887), $4 \mu \mathrm{L}$ CD206-BB515 (clone 19.2, BD564668)) for $30 \mathrm{~min}$ at $4^{\circ} \mathrm{C}$ in the dark. PBMCs were then washed with staining buffer, centrifuged at $250 \boldsymbol{g}$ for $10 \mathrm{~min}$ and resuspended in $250 \mu \mathrm{L}$ fixation/permeabilisation solution (BD Biosciences, San Jose, CA, USA) and placed on ice for $20 \mathrm{~min}$ at $4^{\circ} \mathrm{C}$ in the dark. Hereafter, cells were washed with permeabilisation/wash buffer (BD Biosciences), centrifuged at $250 \mathrm{~g}$ for $10 \mathrm{~min}$ and resuspended in $50 \mu \mathrm{L}$ permeabilisation/wash buffer containing the intracellular antibodies (3.5 $\mu \mathrm{L}$ IL6-APC (clone MQ2-13A5, BD561441), $17 \mu \mathrm{L}$ IL10-PE (BD559330), and $4 \mu \mathrm{L}$ TNFa-PE-Cy7 (BD557647)) and placed at $4^{\circ} \mathrm{C}$ for $30 \mathrm{~min}$ in the dark. Cells were then washed with permeabilisation/wash buffer, centrifuged at $250 \mathrm{~g}$ for $10 \mathrm{~min}$ and resuspended in $500 \mu \mathrm{L}$ staining buffer. This staining procedure allows for the simultaneous detection of surface marker and intracellular cytokine expression. The stain index for each antibody was assessed in titration experiments to determine the optimum concentrations.

Since multicolour cytometric analysis was performed, fluorescent compensation settings were first established through a compensation experiment and regions of positive and negative staining were determined through a fluorochrome minus one experiment. An isotype control cocktail (IgG1-BV421/APC-Cy7/PERCP-Cy5.5/BB5151/APC /PE/PE-Cy7) (Scientific Group, BD 560496, BD560499) 
was used as a negative control for gating purposes. Flow cytometry was performed on the FACS Canto II instrument using FACSDiva software (BD Biosciences). A total of 100,000 events were recorded for each sample. Data analysis was performed using Flow Jo Vx (Treestar, OR, USA) software.

To maintain scientific rigour and to validate the flow cytometry results, the following control treatment groups were assessed for PBMCs derived from a healthy control participant: Unconditioned media; unconditioned media + recombinant IL4 (10 ng/mL; \#574306 Biolegend, San Diego, CA, USA); unconditioned media + recombinant IL6 (400 pg/mL; \#575706 Biolegend); unconditioned media + PAM3CSK4 (100 ng/mL; \#4633/1 R\&D Biosystems Inc); ADSC-CM + anti-IL6 (1:50 rabbit anti-human; \#0002012143 Sigma-Aldrich) and ADSC-CM+anti-PTX3 (1:1000 monoclonal anti-human; \#WH0005806M2 Sigma-Aldrich).

\section{Collection of ADSC-CM}

A human adipose tissue-derived mesenchymal stem cell line (ADSCs) (\#000034977, Poietics, Lonza, Basel, Switzerland) was maintained at $37^{\circ} \mathrm{C}$, in $90 \%$ humidified air with $5 \% \mathrm{CO}_{2}$. ADSCs were cultured in complete media that consisted of DMEM with ultra-glutamine (BioWittaker), containing $1 \%$ penicillin/streptomycin (BioWittaker) and 10\% FBS (Biochrom). For the collection of conditioned media (CM), ADSCs (passages P5-P9) were seeded in T75 flasks (Corning Life Sciences) at a density of $3 \times 10^{5}$ cells in complete medium. After $24 \mathrm{~h}(40 \%$ confluency), $50 \%$ of the CM was removed and replaced with complete medium. After $48 \mathrm{~h}$ (60\% confluency), all the $\mathrm{CM}$ was removed and replaced with fresh complete medium. The ADSCs were allowed to reach confluency, after which cells were sub-cultured and seeded in new T75 flasks for the next round of CM collection. After collection, CM was centrifuged for $10 \mathrm{~min}$ at $1500 \mathrm{~g}$, the pellet containing cellular debris discarded and the supernatant $(\mathrm{CM})$ sterile filtered $(0.2 \mu \mathrm{m})$ (Sigma-Aldrich) before being stored at $-80^{\circ} \mathrm{C}$. To account for intra batch variability, all $\mathrm{CM}$ collected from the different time points and passages were pooled. The cytokine concentration within the CM was analysed using an IL6 ELISA kit (E-EL-H0102, Elabscience Biotechnology Inc.), a CRP kit (E-EL-H5134, Elabscience Biotechnology Inc.) and a 37-Bio-Plex Pro Human inflammation cytokine assay kit (\#171-AL001M, Bio-Rad Laboratories Ltd.). Refer to Table 1 for the concentration of cytokines within the CM.
Table 1 Cytokine concentration within ADSC-CM and assay sensitivity levels.

\begin{tabular}{lccc}
\cline { 1 - 1 } Analyte & Sensitivity $(\mathrm{pg} / \mathrm{mL})$ & & ADSC-CM $(\mathrm{pg} / \mathrm{mL})$ \\
\cline { 1 - 1 } Chitinase-3 like 1 & & 10.3 & 11.8 \\
CRP & $9.38 \times 10^{3}$ & 157.3 \\
IFNB & 2.0 & 17.8 \\
IL6 & 4.69 & 0.6 & 381.5 \\
IL10 & 0.05 & 6.2 \\
IL11 & 1.2 & 635.6 \\
IL26 & 12.3 & 17.5 \\
IL32 & 10.2 & 130.0 \\
LIGHT/TNFSF14 & 0.8 & 94.9 \\
Pentraxin3 & 0.2 & 2218.1 \\
STNFR1 & & 8.3 \\
\hline
\end{tabular}

CRP, C-reactive protein; IFN, interferon; IL, interleukin; LIGHT/TNFSF14, tumour necrosis factor superfamily member 14 ; STNF-R1, soluble tumour necrosis factor receptor.

\section{Statistical analysis}

Data are presented as mean \pm standard error (s.E.). Statistical analysis was performed using Statistica software (version 10, StatSoft, Johannesburg, South Africa). One-way ANOVA with Tukey post hoc test was used to determine group effects, whilst 2-way factorial ANOVAs were used to determine group and treatment effects. The KolmogorovSmirnov (K-S) normality test with Lilliefors correction was used $(P<0.05)$ and normal distribution confirmed with the Shapiro-Wilk test. In cases where data were not normally distributed, non-parametric Kruskal-Wallis ANOVA with Dunns multiple comparison test was used to determine differences between groups, whilst the nonparametric Mann-Whitney test was used to determine the differences between groups and treatment effects. Level of significance was accepted at $P<0.05$.

\section{Results}

\section{Participant demographics and metabolic profiles}

All participants $(n=46)$ were of similar age $(32.3 \pm 1.0$ years $)$, with no difference between groups (Table 2). Refer to Table 2 for detailed information on the lifestyle and nutritional habits of participants. It is noteworthy that the HL group was physically more active (borg scale: $3 \pm 0.3)$ and had good sleeping habits $(90 \%>6 \mathrm{~h}$ per night) but also had a higher percentage of smokers' (70\%) vs non-smokers (30\%) compared to the other groups (Table 2). There was however no difference between the lifestyle and nutritional habits of the OW/OB and MetS groups. Refer to Fig. 1 for detailed information on the body composition and metabolic profile of participants 
Table 2 Lifestyle and nutritional habits of participants.

\begin{tabular}{l}
\hline \\
\hline Age (years) \\
Blood pressure \\
SBP \\
DBP \\
Lifestyle \\
Physical activity (Borg scale) \\
Sleeping (>6 h per night) \\
Average hours per night \\
Smoking \\
Cigarettes per day \\
Alcohol (occasional) \\
Nutritional habits \\
Full meals per day \\
Snacks per day \\
Fruits per week
\end{tabular}

\begin{tabular}{c}
\hline HL $(n=10)$ \\
\hline $29.2 \pm 1.6$ \\
$115.8 \pm 2.6$ \\
$75.2 \pm 1.9$ \\
$3.0 \pm 0.3$ \\
$90.0 \%(9)$ \\
$7.7 \pm 0.4$ \\
$70 \%(7)$ \\
$5.3 \pm 1.5$ \\
$50 \%(5)$ \\
$3.1 \pm 0.3$ \\
$2.0 \pm 1.9$ \\
$5.9 \pm 1.9$
\end{tabular}

\begin{tabular}{c}
\hline OW/OB $(n=22)$ \\
\hline $33.0 \pm 1.5$ \\
$131.0 \pm 3.6^{*}$ \\
$82.6 \pm 2.3$
\end{tabular}

\begin{tabular}{c}
\hline MetS $(n=14)$ \\
\hline $33.5 \pm 1.7$ \\
$132.1 \pm 4.8^{*}$ \\
$89.0 \pm 2.7 *$
\end{tabular}

\begin{tabular}{c} 
Significance \\
\hline NS \\
$P<0.05$ \\
$P<0.01$
\end{tabular}

Values are presented as mean \pm S.E. Physical activity scale: 1-Sedentary; 2-Light activity (no increase in heart rate); 3-Moderate activity (10 min/day); 4-Vigorous activity ( $>1 \mathrm{~h}$ per day on most days). Statistical analysis: One-way ANOVA with Tukey post hoc test.

*Indicates significant difference from HL.

DBP, diastolic blood pressure; NS, non-significant; SBP, systolic blood pressure. Groups: HL, healthy lean; OW/OB, overweight/obese; MetS, metabolic syndrome.

within each group. As expected, the HL group had a normal body composition: BMI $22.1 \pm 0.8 \mathrm{~kg} / \mathrm{m}^{2}$, total body fat: $34.4 \pm 2.3 \%$, trunk-to-limb fat mass ratio (TF/LF): $0.76 \pm 0.06$ and waist-to-hip ratio (WHR): $0.78 \pm 0.02$. This differed significantly from that of the OW/OB and MetS groups $(P<0.05)$ (Fig. 1A, B, C and $\mathrm{D})$. Both the $\mathrm{OW} / \mathrm{OB}$ and MetS groups were obese (BMI: $34.7 \pm 1.6 \mathrm{~kg} / \mathrm{m}^{2}$ vs $\left.32.2 \pm 2.9 \mathrm{~kg} / \mathrm{m}^{2}\right)$ but differed in the percentage of total body fat (OW/OB: $49.7 \pm 1.2 \%$; MetS: $41.3 \pm 1.8 \%, P<0.01$ ) (Fig. $1 \mathrm{~A}$ and $\mathrm{B}$ ) and overall body composition. Visceral adiposity was more pronounced in the MetS group as is evident in the TF/LF ratio (OW/OB: $0.95 \pm 0.04$; MetS: $1.17 \pm 0.05, P<0.01)$ and the WHR (OW/OB: $0.84 \pm 0.01$; MetS: $0.85 \pm 0.02$ ) (Fig. $1 \mathrm{C}$ and D). The DXA scan confirmed fat distribution were predominantly in the subcutaneous regions in the OW/OB group (androidto-gynoid fat mass ratio: $0.93 \pm 0.02$ ) and predominantly in the visceral regions in the MetS group (android-to-gynoid fat mass ratio: $1.04 \pm 0.04$ ) (Fig. 1E).

Metabolic dysregulation was evident in the MetS group, with elevated fasting blood glucose levels (HL: $\quad 5.07 \pm 0.2 \mathrm{mmol} / \mathrm{L} ; \quad$ OW/OB: $\quad 4.9 \pm 0.1 \mathrm{mmol} / \mathrm{L}$; MetS: $7.65 \pm 0.9 \mathrm{mmol} / \mathrm{L}, P<0.001)$, reduced HDL levels (HL: $1.4 \pm 0.08 \mathrm{mmol} / \mathrm{L} ; \quad$ OW/OB: $1.22 \pm 0.05 \mathrm{mmol} / \mathrm{L}$; MetS: $1.05 \pm 0.05 \mathrm{mmol} / \mathrm{L}, \quad P<0.05)$ and elevated TGS (HL: $\quad 0.78 \pm 0.02 \mathrm{mmol} / \mathrm{L} ; \quad \mathrm{OW} / \mathrm{OB}: \quad 0.77 \pm 0.06 \mathrm{mmol} / \mathrm{L}$; MetS: $1.3 \pm 0.21 \mathrm{mmol} / \mathrm{L}, P<0.01$ ) (Fig. $1 F$, G and $\mathrm{H}$ ). No difference was observed between groups with regards to total cholesterol and LDL levels (Fig. 1I and J).

\section{Participant's inflammatory and cytokine profile}

Low-grade systemic inflammation as indicated by elevated serum CRP levels (HL: $136 \pm 40 \mathrm{ng} / \mathrm{mL}$; OW/OB: $\times 11.9$ fold; MetS: $\times 8.5$ fold) were most pronounced in the OW/OB group $(P<0.01)$ (Fig. 2). For all the cytokines assessed in serum, 17 of the analytes were undetectable, 21 analytes could be detected in most of the samples and quantified (Supplementary Table 1) of which only four were significantly different between groups (Fig. 3). Compared to the HL group, obese participants (OW/OB and MetS) had lower levels of IFNa2 (HL $3.3 \pm 0.7 \mathrm{pg} / \mathrm{mL}$; $\mathrm{OW} / \mathrm{OB} \times 0.24$ fold; MetS $\times 0.27$ fold $)(P<0.05)$ (Fig. $3 \mathrm{~A})$, IL12p40 (HL 9.4 $\pm 1.4 \mathrm{pg} / \mathrm{mL}$; OW/OB $\times 0.64$ fold; MetS not detectable) $(P<0.05)$ (Fig. 3B), IL-28A/IFN- $\lambda 2$ (HL $4.7 \pm 1.0 \mathrm{pg} / \mathrm{mL}$; OW/OB $\times 0.45$ fold; MetS $\times 0.47$ fold $)$ $(P<0.05)$ (Fig. 3C), MMP1 (HL $115 \pm 25 \mathrm{pg} / \mathrm{mL}$; OW/OB $\times 0.36$ fold; MetS $\times 0.32$ fold $)(P<0.01)$ (Fig. 3D), MMP3 $(\mathrm{HL} 77 \pm 17 \mathrm{pg} / \mathrm{mL}$; OW/OB $\times 0.7$ fold; MetS $\times 0.51$ fold $)$ $(P<0.05)$ (Fig. 3E) and osteopontin (HL $26 \pm 2 \mathrm{pg} / \mathrm{mL}$; $\mathrm{OW} / \mathrm{OB} \times 0.76$ fold; MetS $\times 0.92$ fold $)(P=0.05)($ Fig. $3 \mathrm{~F})$. No difference was however evident between the cytokine levels of the OW/OB and MetS groups (Fig. 3).

\section{ADSC-CM induced anti-inflammatory response with PBMC populations}

ADSC-CM induced an overall anti-inflammatory phenotype within the entire PBMC population (treatment 

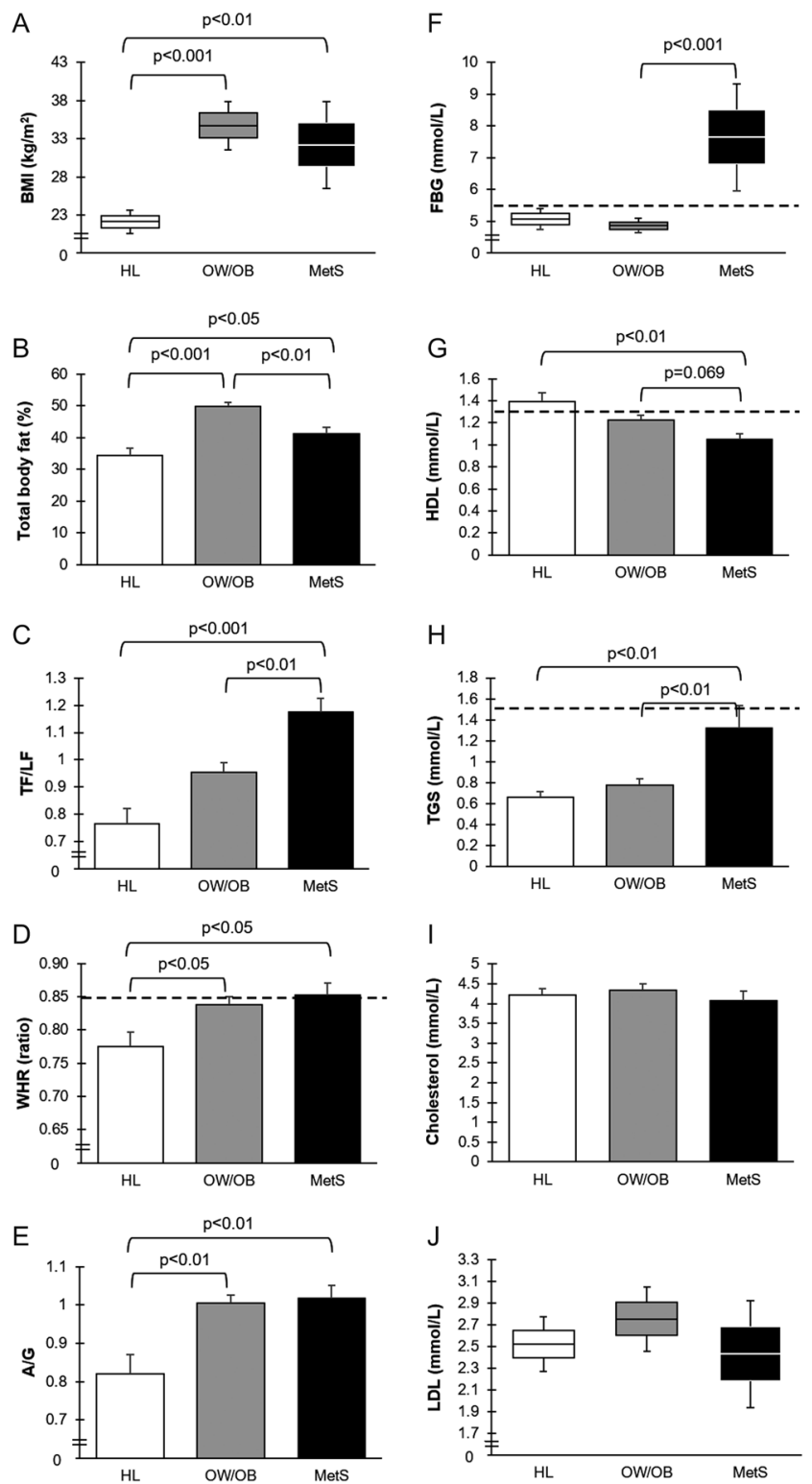

Figure 1

Body composition and metabolic profile of participants within the healthy lean $(n=10)$, overweight/obese $(n=22)$ and metabolic syndrome $(n=14)$ groups. (A) BMI $\left(\mathrm{kg} / \mathrm{m}^{2}\right)$. (B) Total body fat (\%) (df 32). (C) Trunk-to-limb fat mass (TF/LF) ratio (df 32). (D) Waist-to-hip (WHR) ratio (df 32). (E) Android-to-gynoid fat mass (A/G) ratio (df 32). (F) FBG levels (mmol/L). (G) Serum HDL ( $\mathrm{mmol} / \mathrm{L}$ ) (df 43). (H) Serum TGS ( $\mathrm{mmol} / \mathrm{L}$ ) (df 43). (I) Serum cholesterol (mmol/L) (df 43) and (J) Serum LDL (mmol/L). Statistical analysis: One-way ANOVA with Tukey post hoc test (normal distribution), df - degrees of freedom. Non-parametric Kruskal-Wallis ANOVA with Dunns multiple comparison test (data not normally distributed). Level of significance accepted at $P<0.05$. - indicates normal expected physiological range. A/G, android:gynoid ratio; $B M I$, body mass index; $F B G$, fasting blood glucose; HDL, high-density lipoprotein; LDL, low-density lipoprotein; TF/LF, trunk fat:limb fat mass ratio; TGS, triglycerides. Groups: $\mathrm{HL}$, healthy lean group; OW/OB, overweight/obese group; MetS, metabolic syndrome group.

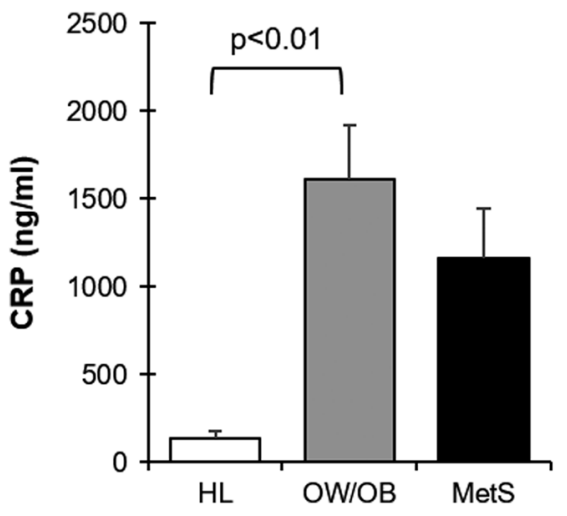

Figure 2

Serum C-reactive protein levels. As indication of systemic inflammation in participants within the healthy lean $(n=10)$, overweight/obese $(n=22)$ and metabolic syndrome $(n=14)$ groups. Statistical analysis: One-Way ANOVA with Tukey post hoc test. Level of significance accepted at $P<0.05, \mathrm{df}=41$. CRP, C-reactive protein. Groups: $\mathrm{HL}$, healthy lean group; OW/OB, overweight/obese group; MetS, metabolic syndrome group.

effect), and although it was most pronounced in the OW/OB group (Fig. 4F), no significant group effect was observed (Fig. 4E, F and G). This is evident in the increased percentage of CD163+ and CD206+positive cells as well as in the increased percentage of cells expressing IL-10 following exposure to ADSC-CM in the OW/OB group compared to the other treatment groups (Fig. 4F). This effect was however blunted, when PBMCs were first exposed to autologous serum in the successive treatment regime (Fig. 4A, B, C, D, E, F and G).

Within the PBMC population, a more detailed investigation into the M1 vs M2 macrophage ratio revealed that ADSC-CM treatment on its own significantly increased the percentage of M2 macrophages compared with the successive treatment (Fig. 5B, E, F and G), whilst reducing the percentage of $\mathrm{M} 1$ macrophages (Fig. 5B, E, F and G). Priming the PBMCs with autologous serum (prior to treatment) increased M1 and reduced M2 macrophages (Fig. 5E, $F$ and $G$ ), suggesting that longer ADSC-CM treatment periods might be necessary to induce the anti-inflammatory switch. The successive treatment is therefore not a recommended strategy.

Very few of the naïve/unstimulated M1 macrophages were positive for the intracellular cytokines (IL6, IL10 and TNFa) (Fig. 5C). However, upon stimulation with either unconditioned culture media or an activating agent (Pam3CSK4), these M1 macrophages expressed both IL6 and TNFa (Fig. 5D), confirming their inflammatory phenotype. Both the successive treatment regime and ADSC-CM on its own increased the intracellular expression 

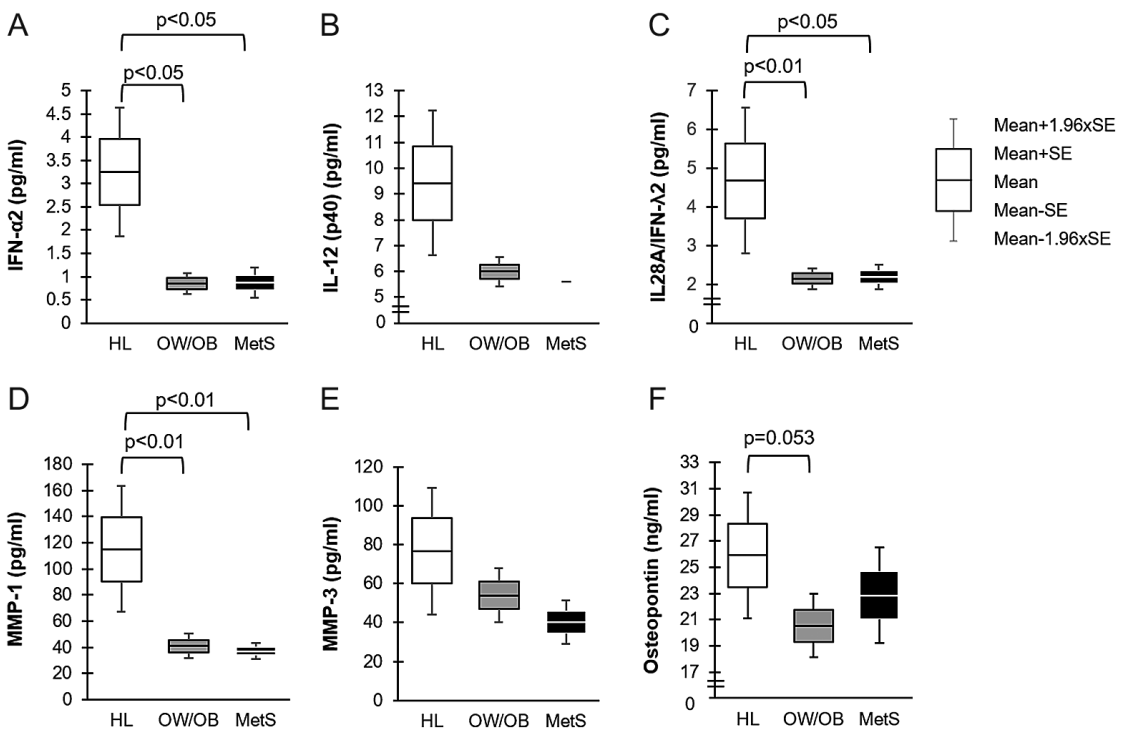

F

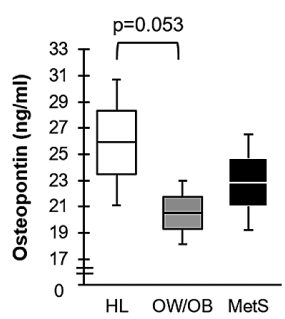

\section{Figure 3}

Inflammatory cytokine profile of participants within the healthy lean $(n=10)$, overweight/obese $(n=22)$ and metabolic syndrome $(n=14)$ groups. Serum concentrations of cytokines that differed between groups are presented as box-andwhisker plots (data not normally distributed) (A) IFNa2 (pg/mL), (B) IL12 (p40) (pg/mL), (C) IL28A/IFN- $\lambda 2$ (pg/mL), (D) MMP1 (pg/mL), (E) MMP3 (pg/mL) and (F) Osteopontin ( $\mathrm{ng} / \mathrm{mL})$. Statistical analysis: Non-parametric Kruskal-Wallis ANOVA with Dunns multiple comparisons test or Mann-Whitney test (B). IFN, interferon; IL, interleukin; MMP, matrix metalloproteinase. Groups: $\mathrm{HL}$, healthy lean group; OW/OB, overweight/obese group; MetS, metabolic syndrome group. of IL10 within the M2 macrophages compared to exposure with autologous serum (Fig. 6A, B and C). The ADSC-CMinduced expression of IL10 was even more pronounced in the activated M2 macrophage population of the OW/ $\mathrm{OB}$ and MetS groups (Fig. 6B and C). Successive treatment did however have a greater effect on intracellular IL6 expression within the inactive M2 macrophages than ADSC-CM treatment on its own (Fig. 6D, E and F). Intracellular TNFa expression within M2 macrophages was not affected by treatment (Fig. 6G, H and I).
A

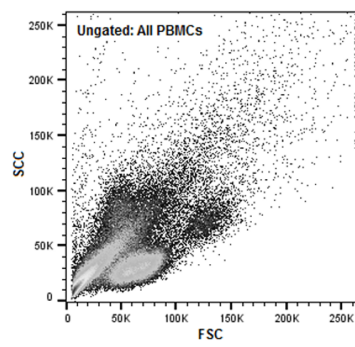

E

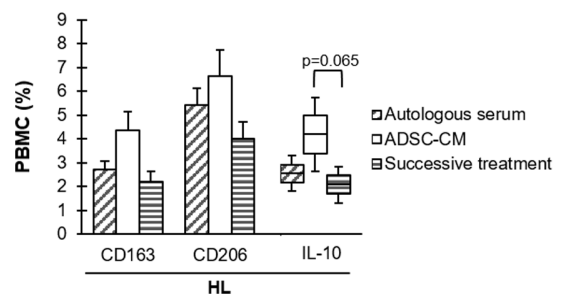

B

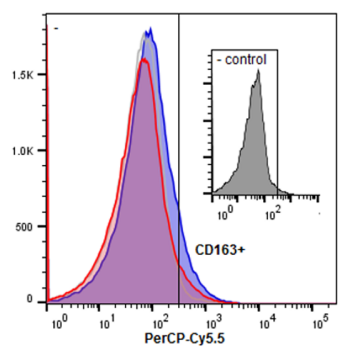

$\mathrm{F}$
C
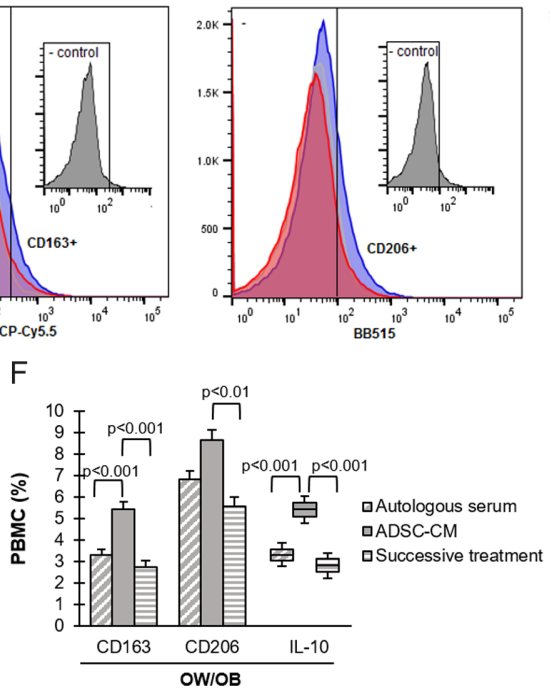

D

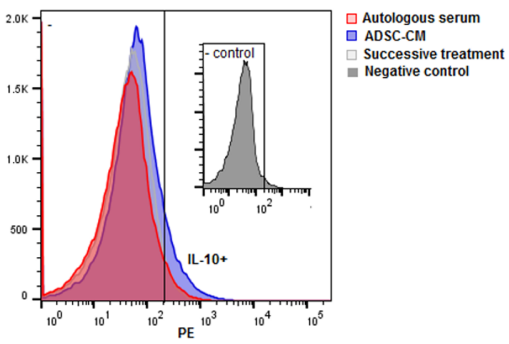

G

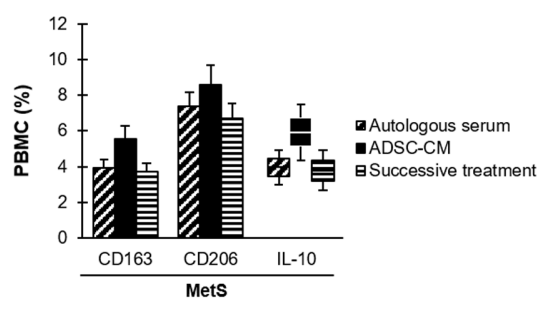

Figure 4

The responsiveness of the overall participant-derived PBMC population to ADSC-CM treatment. (A) Forward vs Side scatter (ungated) indicating the size vs granularity of the PBMC populations. (B, $C$ and $D$ ) Representative flow cytometry histograms indicating regions of positive and negative staining for CD 163-PerCP-Cy5.5 (B), CD206-BB515 (C) and IL10-PE (D). (E, F and G) The percentage of PBMCs positive for CD163, CD206 and IL10 following autologous serum, ADSC-CM or successive treatments within each group (HL $n=10$; OW/OB $n=22$; MetS $n=14)$. Statistical analysis: Non-parametric Kruskal Wallis ANOVA with Dunns multiple comparisons test (IL-10) or factorial ANOVA with Tukey post hoc test (CD163 and CD206) df $=129$. CD, cluster of differentiation; FSC, forward scatter; IL, interleukin; PBMCs, peripheral blood mononuclear cells; SCC, side scatter. Groups: HL, healthy lean group; OW/ $\mathrm{OB}$, overweight/obese group; MetS, metabolic syndrome group. Treatments: ADSC-CM, adipose-derived stem cell conditioned media. A full colour version of this figure is available at https://doi.org/10.1530/JME-18-0078. 

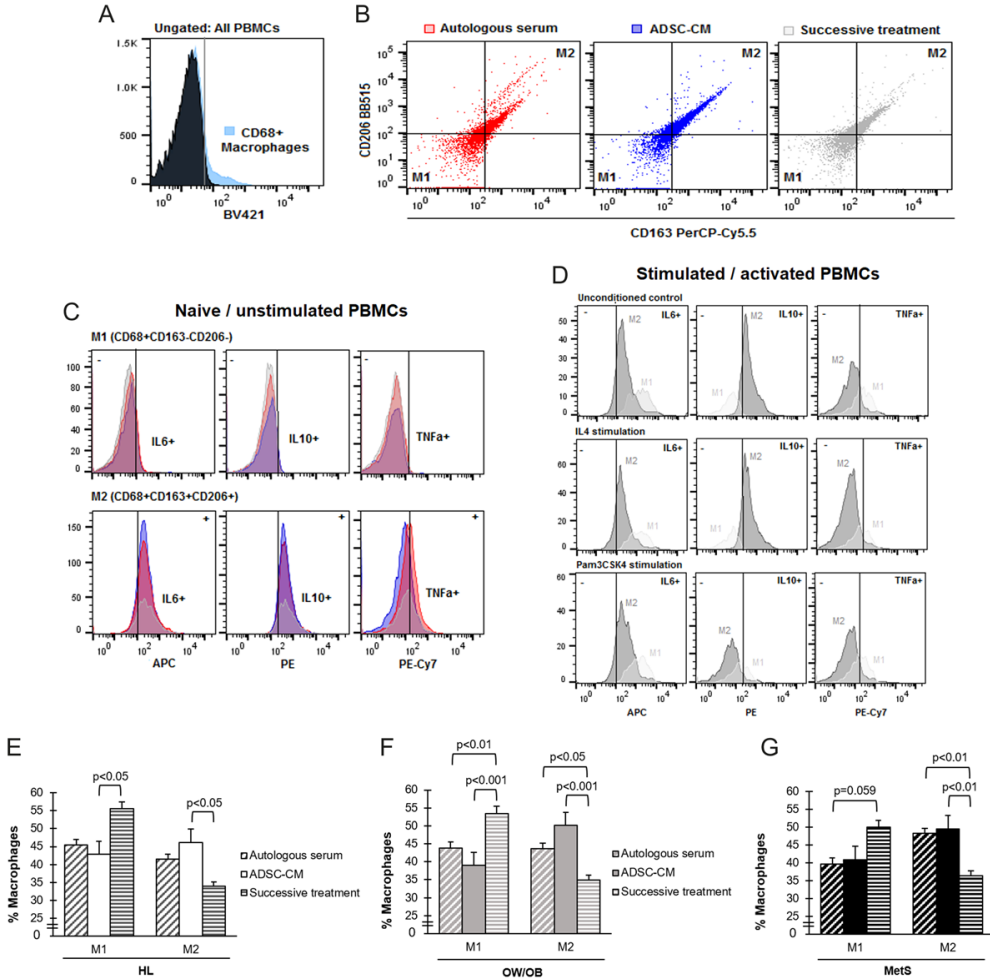

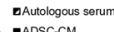
ADSC.CM

\section{Figure 5}

The responsiveness of CD68+ macrophages present within the participant-derived PBMCs to ADSC-CM treatment. (A) Representative flow cytometry histogram indicating regions of positive and negative staining for CD68-BV421 within the PBMC population. (B) Scatter plots indicating the ratio of M1 (CD206-CD163-) vs M2 (CD206+CD163+) macrophages within the CD68+ gated population following either autologous serum, ADSC-CM or successive treatment. (C) Representative flow cytometry histograms indicating intracellular cytokine (IL-6-APC, IL-10-PE and TNF- $\alpha$-PE-Cy7) expression in naïve/unstimulated M1 (CD68+CD163-CD206-) or M2 (CD68+CD163+CD206+) macrophages following treatment. (D) Representative flow cytometry histograms indicating intracellular cytokine expression (IL-6-APC, IL-10-PE and TNF- $\alpha$-PE-Cy7) upon stimulation with either unconditioned media, recombinant IL4 or Pam3CSK4. (E, F and G) Quantification of the percentage of M1 and M2 macrophages in the HL $(n=10)$, OW/OB $(n=22)$ and MetS $(n=14)$ groups following each treatment regime. Statistical analysis: Factorial ANOVA with Tukey post hoc test $d f=129 . C D$ cluster of differentiation; IL, interleukin; PBMCs, peripheral blood mononuclear cells; TNF, tumour necrosis factor. Groups: $\mathrm{HL}$, healthy lean group; OW/OB, overweight/obese group; MetS, metabolic syndrome group. Treatments: ADSC-CM, adipose-derived stem cell conditioned media. A full colour version of this figure is available at https://doi.org/10.1530/JME-18-0078.
rhIL6 treatment induced IL10 expression in M2 macrophages to a similar extent than ADSC-CM, however, blocking either IL6 or PTX3 does not blunt the IL10 response

The treatment of healthy lean control PBMCs with rhIL6 (400 pg/mL; comparable to the concentration detected in ADSC-CM $381 \mathrm{pg} / \mathrm{mL}$ ), induced the expression of intracellular IL10 within M2 macrophages compared to unconditioned control media (Fig. 7A). Treatment of ADSC-CM induced a similar response (Fig. 7B), and blocking of either IL6 or PTX3 within ADSC-CM during treatment did not inhibit the IL10 response (Fig. 7C and D).

\section{Discussion}

Pathological changes within the systemic microenvironment during obesity and metabolic syndrome modulates the function and gene expression profile of PBMCs (Manoel-Caetano et al. 2012, de Luis et al. 2017). Failure of anti-inflammatory control mechanisms together with a skewed ratio of pro- (M1) vs anti- (M2) inflammatory macrophages within peripheral circulation has been associated with the metabolic complications of obesity and disease progression (Bories et al. 2012, Finucane et al. 2012, Rentoukas et al. 2012, Catalán et al. 2015). Although MSCs are known to play a role in the immune modulation of inflammatory responses (Kwon et al. 2014, Zimmermann \& McDevitt 2014), this study is the first to demonstrate the therapeutic potential of ADSC-derived conditioned media to restore the inflammatory balance in immune compromised obese individuals. It should however be noted that the physical activity, smoking and sleeping habits of the healthy lean participants differed from the other groups and could be contributing factors. Even though this study was done in an ex vivo setting and needs validation in vivo, it signified that although obesity per se (not dyslipidaemia or glucose dysregulation) is the main cause of inflammatory dysregulation, PBMCs are more responsive to conditioned media treatment prior to the development of metabolic syndrome.

The clinical data in this study clearly demonstrated dyslipidaemia (reduced HDL, elevated TGS) and glucose dysregulation (elevated FBG) in the MetS group that was not evident in the $\mathrm{OW} / \mathrm{OB}$ group. Despite this metabolic dysregulation, no difference was detected in 
A

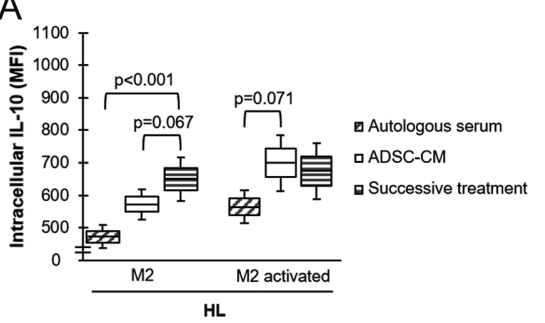

D
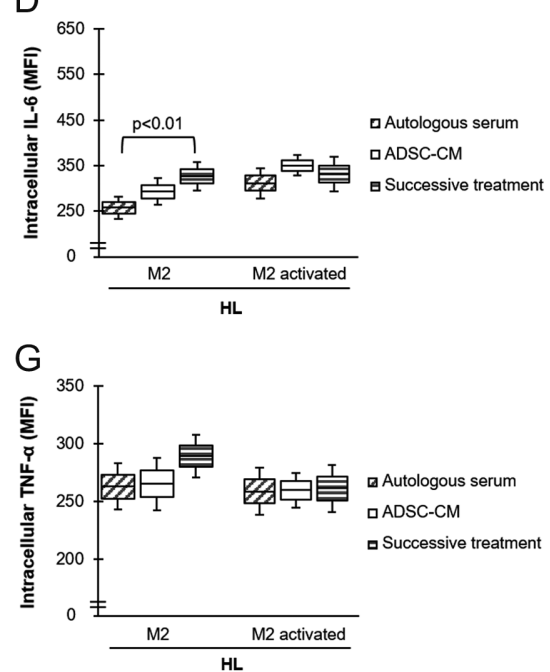

B

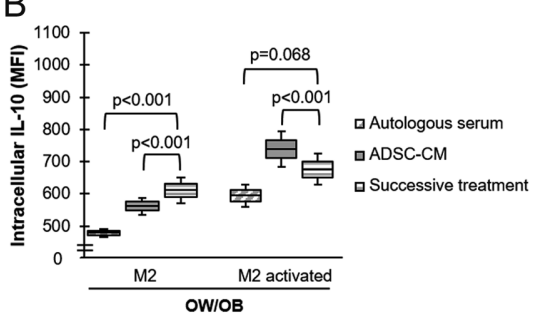

$E$

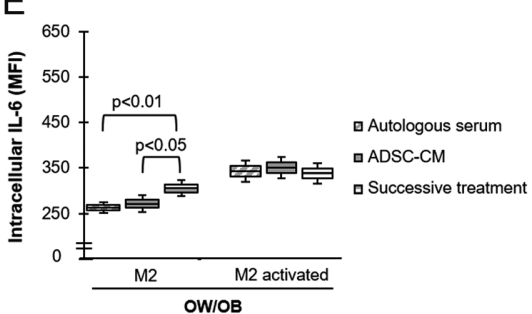

$\mathrm{H}$

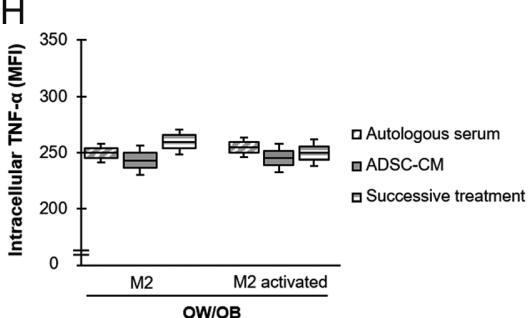

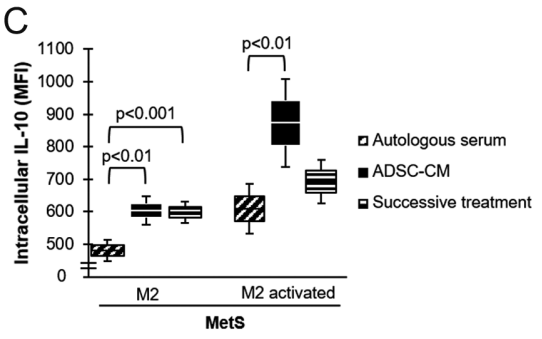

$\mathrm{F}$

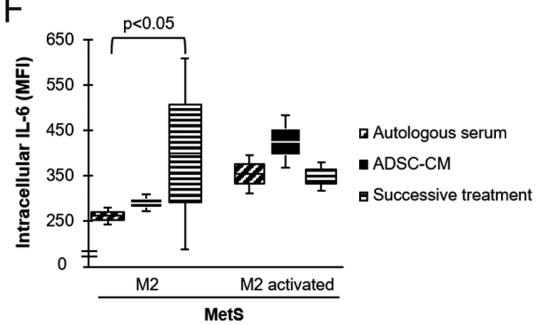

I

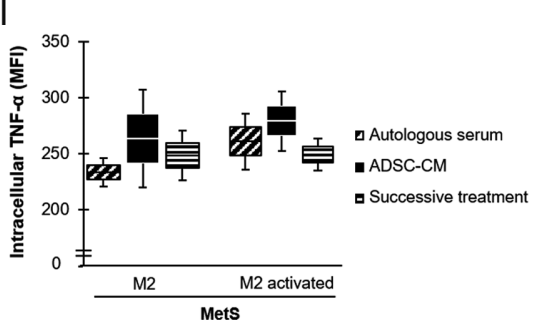

Figure 6

The intracellular cytokine expression within the M2 population following exposure to ADSC-CM. M2 macrophages (CD68+CD206+CD163+) were regarded as activated when they also expressed the activation marker CD11 b+. (A, B and C) Intracellular IL-10 expression, (D, E and F) intracellular IL-6 expression, (G, H and I) intracellular TNF- $\alpha$ expression. Statistical analysis: Non-parametric Kruskal Wallis ANOVA with Dunns multiple comparisons test. $\mathrm{IL}$, interleukin; MFI, mean fluorescent intensity; TNF, tumour necrosis factor. Groups: $\mathrm{HL}$, healthy lean group; OW/OB, overweight/obese group; MetS, metabolic syndrome group. Treatments: ADSC-CM, adipose-derived stem cell conditioned media.

the serum cytokine profiles of these two groups. The obese participants (regardless of metabolic syndrome) all had systemic inflammation (elevated CRP levels) and reduced serum levels of IFNa2, IL12p40 and IFN/IL28A indicative of suppressed immunity (Terán-Cabanillas et al. 2014). This is in agreement with numerous reports demonstrating compromised viral and host responses during obesity (Tominaga et al. 2010, TeranCabanillas et al. 2013, 2014). This study furthermore demonstrated a reduced level of circulating MMP1. Matrix metalloproteinases, such as MMP1, plays a role in adipose tissue remodelling during obesity, and it has been shown that macrophage derived factors can modulate the secretion of MMPs by adipocytes (O'Hara et al. 2009, Gao \& Bing 2011). Gao et al. (2017) demonstrated that macrophage accumulation in adipose tissue stimulates the production of MMP1 and MMP3 and that inhibition of IL1B in vitro abolishes the production of these MMPs by adipocytes. In agreement with our study, Song et al. (2018) recently observed a reduction in MMP1 tissue levels during obesity in mice following a high-fat diet. Taken together, these studies highlight the central role of macrophages in obesity and metabolic disease.

Targeting PBMCs with conditioned media derived from healthy-donor ADSCs elicited a similar antiinflammatory response in all participants, the PBMCs from OW/OB participants were however slightly more responsive to treatment than those derived from participants with metabolic syndrome. Within PBMCs derived from the OW/OB group, conditioned media treatment increased the percentage of cells that expressed CD163, CD206 and the anti-inflammatory cytokine IL10. This is consistent with previous findings demonstrating that co-culture of PBMCs and MSCs do not enhance the activation status of unstimulated PBMCs but instead perform supportive functions (Gornostaeva et al. 2018). The macrophage fraction of the PBMCs derived from both OW/OB and MetS participants responded in a similar fashion to conditioned media treatment by increasing 
A

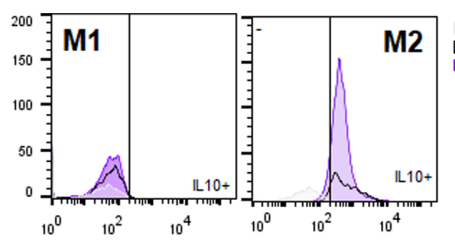

B

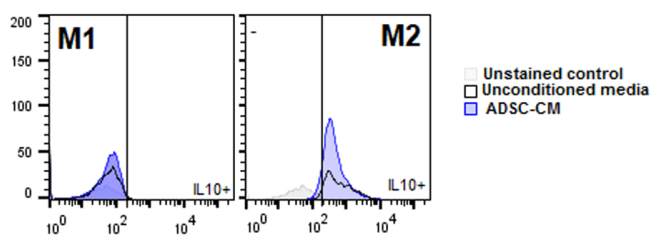

C
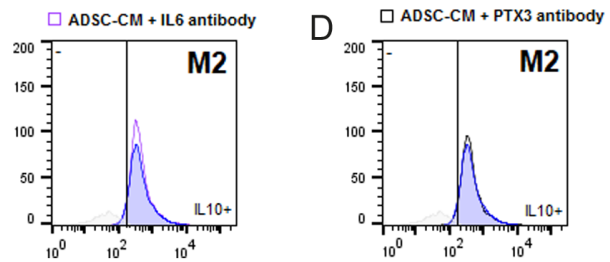

Figure 7

The intracellular IL10 expression within the M2 population following exposure to (A) recombinant IL6 $(400 \mathrm{pg} / \mathrm{mL})$ and (B) ADSC-CM compared to treatment with unconditioned control media. ( $C$ and $D)$ Intracellular M2 IL10 expression following ADSC-CM treatment with either anti-IL6 blocking antibody (C) or anti-PTX3 blocking antibody (D) compared to ADSC-CM treatment. Representative flow cytometry histograms are from a randomly selected healthy lean participant. A full colour version of this figure is available at https://doi.org/10.1530/JME-18-0078.

the production of intracellular IL10. It is consistent with previous observations demonstrating that co-culture of PBMCs with either embryonic or bone marrow-derived MSCs induce the production of IL10 (Conforti et al. 2014, Lotfinia et al. 2017). In vivo, this anti-inflammatory response would dampen the phagocytic and destructive effects of M1 macrophages in order to create a growthpromoting microenvironment that favours regeneration and remodelling. It is thus possible that conditioned media treatment could potentially counteract systemic inflammation in high-risk patients through antiinflammatory control mechanisms.

The ADSC-derived conditioned media contained high concentrations of the IL6 superfamily of cytokines (IL6 and IL11) as well as the pattern recognising protein, Pentraxin3 (PTX3). Overexpression of PTX3 in human THP1 macrophages have been shown to increase oxidative LDL uptake and reduce cholesterol efflux in macrophage foam cells (Liu et al. 2014), implicating a role for PTX3 in atherosclerosis (Vilahur \& Badimon 2015). Recent studies have however shown that PTX3 is an anti-inflammatory protein that regulates the inflammatory activity of macrophages and therefore has athero-protective properties (Shiraki et al. 2016, Slusher et al. 2016). Slusher et al. (2016) indicated that lipid (palmitate)-induced expression of PTX3 in PBMCs derived from healthy men was associated with an increase in the production of IL10. This is consistent with our observations and suggests that PTX3 together with IL6 (known to upregulate IL10 production) within conditioned media were responsible for modulating the phenotype and cytokine responses of PBMCs/macrophages. Treatment of PBMCs with recombinant IL6 furthermore induced the expression of IL10 in M2 macrophages and supports this hypothesis. However, when either IL6 or PTX3 was blocked in ADSC-CM, the IL10 response remained unaffected. Taken together, it suggests that there are likely numerous unidentified complementary factors within ADSC-CM capable of inducing an anti-inflammatory response. The therapeutic potential of ADSCs-derived conditioned media is thus promising and needs validation in the clinical setting.

\section{Supplementary data}

This is linked to the online version of the paper at https://doi.org/10.1530/ JME-18-0078.

Declaration of interest

The authors declare that there is no conflict of interest that could be perceived as prejudicing the impartiality of the research reported.

\section{Funding}

This study was supported by the Faculty of Medicine and Health Sciences (Early career research grant), Stellenbosch University and the South African Medical Research Council (SAMRC) under a Self-Initiated Research Grant. Even though the work is supported by the SAMRC, the views and opinions expressed are not those of the SAMRC but of the authors. M J Kruger was supported by a postdoctoral fellowship from the Faculty of Medicine and Health Sciences, Stellenbosch University.

\section{Acknowledgements}

The authors of this study would like to thank Srs Leandra Pedro and Francis Pietersen for their assistance in the collection of blood samples.

\section{References}

Boland L, Burand AJ, Brown AJ, Boyt D, Lira VA \& Ankrum JA 2017 IFN$\gamma$ and TNF- $\alpha$ pre-licensing protects mesenchymal stromal cells from the pro-inflammatory effects of palmitate. Molecular Therapy $\mathbf{2 6}$ 860-873. (https://doi.org/10.1016/j.ymthe.2017.12.013)

Bories G, Caiazzo R, Derudas B, Copin C, Raverdy V, Pigeyre M, Pattou F, Staels B \& Chinetti-Gbaguidi G 2012 Impaired alternative macrophage differentiation of peripheral blood mononuclear cells 
from obese subjects. Diabetes and Vascular Disease Research 9 189-195. (https://doi.org/10.1177/1479164111430242)

Braune J, Weyer U, Hobusch C, Mauer J, Brüning JC, Bechmann I \& Gericke M 2017 IL-6 regulates M2 polarization and local proliferation of adipose tissue macrophages in obesity. Journal of Immunology 198 2927-2934. (https://doi.org/10.4049/ jimmunol.1600476)

Catalán V, Gómez-Ambrosi J, Rodríguez A, Ramírez B, Valentí V, Moncada R, Silva C, Salvador J \& Frühbeck G 2015 Peripheral mononuclear blood cells contribute to the obesity-associated inflammatory state independently of glycemic status: involvement of the novel proinflammatory adipokines chemerin, chitinase-3-like protein 1, lipocalin-2 and osteopontin. Genes and Nutrition 10460 (https://doi.org/10.1007/s12263-015-0460-8)

Chen W, Huang Y, Han J, Yu L, Li Y, Lu Z, Li H, Liu Z, Shi C, Duan F, et al. 2016 Immunomodulatory effects of mesenchymal stromal cellsderived exosome. Immunologic Research 64 831-840. (https://doi. org/10.1007/s12026-016-8798-6)

Conforti A, Scarsella M, Starc N, Giorda E, Biagini S, Proia A, Carsetti R, Locatelli F \& Bernardo ME 2014 Microvescicles derived from mesenchymal stromal cells are not as effective as their cellular counterpart in the ability to modulate immune responses in vitro. Stem Cells and Development 23 2591-2599. (https://doi.org/10.1089/ scd.2014.0091)

de Luis DA, Almansa R, Aller R, Izaola O \& Romero E 2017 Gene expression analysis identify a metabolic and cell function alterations as a hallmark of obesity without metabolic syndrome in peripheral blood, a pilot study. Clinical Nutrition 37 1348-1353. (https://doi. org/10.1016/j.clnu.2017.06.006)

Dey A, Allen J \& Hankey-Giblin PA 2014 Ontogeny and polarization of macrophages in inflammation: blood monocytes versus tissue macrophages. Frontiers in Immunology 5 683. (https://doi. org/10.3389/fimmu.2014.00683)

Egan CG, Lavery R, Caporali F, Fondelli C, Laghi-Pasini F, Dotta F \& Sorrentino V 2008. Generalised reduction of putative endothelial progenitors and CXCR4-positive peripheral blood cells in type 2 diabetes. Diabetologia 51 1296-1305. (https://doi.org/10.1007/ s00125-008-0939-6)

Esser N, Legrand-Poels S, Piette J, Scheen AJ \& Paquot N 2014 Inflammation as a link between obesity, metabolic syndrome and type 2 diabetes. Diabetes Research and Clinical Practice 105 141-150. (https://doi.org/10.1016/j.diabres.2014.04.006)

Fadini GP, Ciciliot S \& Albiero M 2017 Concise review: perspectives and clinical implications of bone marrow and circulating stem cell defects in diabetes. Stem Cells 35 106-116. (https://doi.org/10.1002/stem.2445)

Finucane OM, Reynolds CM, McGillicuddy FC \& Roche HM 2012 Insights into the role of macrophage migration inhibitory factor in obesity and insulin resistance. Proceedings of the Nutrition Society $\mathbf{7 1}$ 622-633. (https://doi.org/10.1017/S0029665112000730)

Fontaine MJ, Shih H, Schäfer R \& Pittenger MF 2016 Unraveling the mesenchymal stromal cells' paracrine immunomodulatory effects. Transfusion Medicine Reviews 30 37-43. (https://doi.org/10.1016/j. tmrv.2015.11.004)

Gao D \& Bing C 2011 Macrophage-induced expression and release of matrix metalloproteinase 1 and 3 by human preadipocytes is mediated by IL-1 $\beta$ via activation of MAPK signaling. Journal of Cellular Physiology 226 2869-2880. (https://doi.org/10.1002/ jcp.22630)

Gao X, Salomon C \& Freeman DJ 2017 Extracellular vesicles from adipose tissue - a potential role in obesity and type 2 diabetes? Frontiers in Endocrinology 8 202. (https://doi.org/10.3389/ fendo.2017.00202)

Goedecke JH, Mtintsilana A, Dlamini SN \& Kengne AP 2017 Type 2 diabetes mellitus in African women. Diabetes Research and Clinical Practice 123 87-96. (https://doi.org/10.1016/j.diabres.2016.11.017)
Gornostaeva AN, Andreeva ER, Bobyleva PI \& Buravkova LB 2018 Interaction of allogeneic adipose tissue-derived stromal cells and unstimulated immune cells in vitro: the impact of cell-to-cell contact and hypoxia in the local milieu. Cytotechnology 70 299-312. (https:// doi.org/10.1007/s10616-017-0144-x)

Harting MT, Srivastava AK, Zhaorigetu S, Bair H, Prabhakara KS, Toledano Furman NE, Vykoukal JV, Ruppert KA, Cox CS \& Olson SD 2018 Inflammation-stimulated mesenchymal stromal cell-derived extracellular vesicles attenuate inflammation. Stem Cells 36 79-90. (https://doi.org/10.1002/stem.2730)

Kwon M-S, Noh M-Y, Oh K-W, Cho K-A, Kang B-Y, Kim K-S, Kim Y-S \& Kim SH 2014 The immunomodulatory effects of human mesenchymal stem cells on peripheral blood mononuclear cells in ALS patients. Journal of Neurochemistry 131 206-218. (https://doi. org/10.1111/jnc.12814)

Liu W, Jiang J, Yan D, Li D, Li W, Ma Y, Yang L, Qu Z \& Ruan Q 2014 Pentraxin 3 promotes oxLDL uptake and inhibits cholesterol efflux from macrophage-derived foam cells. Experimental and Molecular Pathology 96 292-299. (https://doi.org/10.1016/j.yexmp. 2014.03.007)

Lotfinia M, Lak S, Mohammadi Ghahhari N, Johari B, Maghsood F, Parsania S, Sadegh Tabrizi B \& Kadivar M 2017 Hypoxia preconditioned embryonic mesenchymal stem cell secretome reduces IL-10 production by peripheral blood mononuclear cells. Iranian Biomedical Journal 21 24-31.

Manoel-Caetano FS, Xavier DJ, Evangelista AF, Takahashi P, Collares CV, Puthier D, Foss-Freitas MC, Foss MC, Donadi EA, Passos GA, et al. 2012 Gene expression profiles displayed by peripheral blood mononuclear cells from patients with type 2 diabetes mellitus focusing on biological processes implicated on the pathogenesis of the disease. Gene 511 151-160. (https://doi.org/10.1016/j. gene.2012.09.090)

Martyniak K \& Masternak MM 2017 Changes in adipose tissue cellular composition during obesity and aging as a cause of metabolic dysregulation. Experimental Gerontology 94 59-63. (https://doi. org/10.1016/j.exger.2016.12.007)

O'Hara A, Lim F-L, Mazzatti DJ \& Trayhurn P 2009 Microarray analysis identifies matrix metalloproteinases (MMPs) as key genes whose expression is up-regulated in human adipocytes by macrophageconditioned medium. Pflugers Archiv: European Journal of Physiology 458 1103-1114. (https://doi.org/10.1007/s00424-009-0693-8)

Pirola L \& Ferraz JC 2017 Role of pro- and anti-inflammatory phenomena in the physiopathology of type 2 diabetes and obesity. World Journal of Biological Chemistry 8 120-128. (https://doi. org/10.4331/wjbc.v8.i2.120)

Ra JC, Kang SK, Shin IS, Park HG, Joo SA, Kim JG, Kang B-C, Lee YS, Nakama K, Piao M, et al. 2011a Stem cell treatment for patients with autoimmune disease by systemic infusion of culture-expanded autologous adipose tissue derived mesenchymal stem cells. Journal of Translational Medicine 9 181. (https://doi.org/10.1186/1479-5876-9181)

Ra JC, Shin IS, Kim SH, Kang SK, Kang BC, Lee HY, Kim YJ, Jo JY, Yoon EJ, Choi HJ, et al. 2011b Safety of intravenous infusion of human adipose tissue-derived mesenchymal stem cells in animals and humans. Stem Cells and Development 20 1297-1308. (https://doi. org/10.1089/scd.2010.0466)

Rentoukas E, Tsarouhas K, Kaplanis I, Korou E, Nikolaou M, Marathonitis G, Kokkinou S, Haliassos A, Mamalaki A, Kouretas D, et al. 2012 Connection between telomerase activity in PBMC and markers of inflammation and endothelial dysfunction in patients with metabolic syndrome. PLOS ONE 7 e35739. (https://doi. org/10.1371/journal.pone.0035739)

Rigato M \& Fadini GP 2017 Circulating stem/progenitor cells as prognostic biomarkers in macro- and microvascular disease. A narrative review of prospective observational studies. Current 
Medicinal Chemistry Epub. (https://doi.org/10.2174/092986732466617 0920154020)

Shiraki A, Kotooka N, Komoda H, Hirase T, Oyama J-I \& Node K 2016 Pentraxin-3 regulates the inflammatory activity of macrophages. Biochemistry and Biophysics Reports 5 290-295. (https://doi. org/10.1016/j.bbrep.2016.01.009)

Slusher AL, Mischo AB \& Acevedo EO 2016 Pentraxin 3 is an antiinflammatory protein associated with lipid-induced interleukin 10 in vitro. Cytokine 86 36-40. (https://doi.org/10.1016/j.cyto. 2016.07.012)

Song B, Zhang H \& Zhang S 2018 Toll-like receptor 2 mediates deposition of collagen I in adipose tissue of high fat diet-induced obese mice. Molecular Medicine Reports 17 5958-5963. (https://doi. org/10.3892/mmr.2018.8590)

Teran-Cabanillas E, Montalvo-Corral M, Caire-Juvera G, MoyaCamarena SY \& Hernández J 2013 Decreased interferon- $\alpha$ and interferon- $\beta$ production in obesity and expression of suppressor of cytokine signaling. Nutrition 29 207-212. (https://doi.org/10.1016/j. nut.2012.04.019)

Terán-Cabanillas E, Montalvo-Corral M, Silva-Campa E, Caire-Juvera G, Moya-Camarena SY \& Hernández J 2014 Production of interferon $\alpha$ and $\beta$, pro-inflammatory cytokines and the expression of suppressor of cytokine signaling (SOCS) in obese subjects infected with influenza A/H1N1. Clinical Nutrition 33 922-926. (https://doi. org/10.1016/j.clnu.2013.10.011)
Tominaga M, Uno K, Yagi K, Fukui M, Hasegawa G, Yoshikawa T, Nakamura N \& Nakumura N 2010 Association between capacity of interferon-alpha production and metabolic parameters. Journal of Interferon and Cytokine Research 30 451-454. (https://doi.org/10.1089/ jir.2009.0085)

Toupet K, Maumus M, Peyrafitte J-A, Bourin P, van Lent PLEM, Ferreira R, Orsetti B, Pirot N, Casteilla L, Jorgensen C, et al. 2013 Long-term detection of human adipose-derived mesenchymal stem cells after intraarticular injection in SCID mice. Arthritis and Rheumatism 65 1786-1794. (https://doi.org/10.1002/art.37960)

van de Vyver M 2017 Intrinsic mesenchymal stem cell dysfunction in diabetes mellitus: implications for autologous cell therapy. Stem Cells and Development 26 1042-1053. (https://doi.org/10.1089/ scd.2017.0025)

Vilahur G \& Badimon L 2015 Biological actions of pentraxins. Vascular Pharmacology 73 38-44. (https://doi.org/10.1016/j. vph.2015.05.001)

Zachar L, Bačenková D \& Rosocha J 2016 Activation, homing, and role of the mesenchymal stem cells in the inflammatory environment. Journal of Inflammation Research 9 231-240. (https://doi.org/10.2147/ JIR.S121994)

Zimmermann JA \& McDevitt TC 2014 Pre-conditioning mesenchymal stromal cell spheroids for immunomodulatory paracrine factor secretion. Cytotherapy 16 331-345. (https://doi.org/10.1016/j. jcyt.2013.09.004)

Received in final form 9 July 2018

Accepted 23 July 2018

Accepted Preprint published online 23 July 2018 (c) 2018 Society for Endocrinology Published by Bioscientifica Ltd. Printed in Great Britain 